Research Article

\title{
Higher-Order Uniformly Convergent Numerical Scheme for Singularly Perturbed Differential Difference Equations with Mixed Small Shifts
}

\author{
Mesfin Mekuria Woldaregay $\mathbb{i D}^{1}$ and Gemechis File Duressa $\mathbb{D}^{2}$ \\ ${ }^{1}$ Department of Applied Mathematics, Adama Science and Technology University, Adama, Ethiopia \\ ${ }^{2}$ Department of Mathematics, Jimma University, Jimma, Ethiopia
}

Correspondence should be addressed to Mesfin Mekuria Woldaregay; msfnmkr02@gmail.com

Received 31 October 2020; Revised 4 December 2020; Accepted 12 December 2020; Published 28 December 2020

Academic Editor: Andrea Scapellato

Copyright (c) 2020 Mesfin Mekuria Woldaregay and Gemechis File Duressa. This is an open access article distributed under the Creative Commons Attribution License, which permits unrestricted use, distribution, and reproduction in any medium, provided the original work is properly cited.

\begin{abstract}
This paper deals with numerical treatment of singularly perturbed differential difference equations involving mixed small shifts on the reaction terms. The highest-order derivative term in the equation is multiplied by a small perturbation parameter $\varepsilon$ taking arbitrary values in the interval $(0,1]$. For small values of $\varepsilon$, the solution of the problem exhibits exponential boundary layer on the left or right side of the domain and the derivatives of the solution behave boundlessly large. The terms having the shifts are treated using Taylor's series approximation. The resulting singularly perturbed boundary value problem is solved using exponentially fitted operator FDM. Uniform stability of the scheme is investigated and analysed using comparison principle and solution bound. The formulated scheme converges uniformly with linear order before Richardson extrapolation and quadratic order after Richardson extrapolation. The theoretical analysis of the scheme is validated using numerical test examples for different values of $\varepsilon$ and mesh number $N$.
\end{abstract}

\section{Introduction}

Differential equations play a prominent role in many disciplines including engineering, physics, economics, and biology. Currently different authors are working on analytical and numerical solutions of differential equations using different techniques [1, 2]. Differential difference equations (DDEs) are differential equations where the evolution of the system not only depends on the present state of the system but also depends on the past history. Singularly perturbed differential difference equations are differential equations in which the highest-order derivative term is multiplied by a small perturbation parameter $\varepsilon$ and involves at least one term with delay. In general, when the perturbation parameter tends to zero, the smoothness of the solution of the singularly perturbed differential difference equations (SPDDEs) deteriorates and it forms boundary layer [3]. Such type of equations has applications in the study of variational problems in control theory [4] and in modelling of neuronal variability [5].

The presence of singular perturbation parameter $\varepsilon$ in the equation leads to oscillation in the computed solution, while using standard numerical methods like FDM, FEM, and spline method [6]. To avoid this oscillation, an unacceptably large number of mesh points are required when $\varepsilon$ is very small. This is not practical and leads to round-off error. So, to overcome the drawbacks associated with standard numerical methods, different authors have developed schemes that converge uniformly.

Numerical treatments of a class of SPDDEs have received a great deal of attention recently because of their wide applications. It is of theoretical and practical interest to consider numerical methods for such problems. Owing to this, here we present some prior studies on numerical solution of the considered problem. Lange and Miura in [7-10] studied a class of second-order DDEs in which the second 
derivative term is multiplied by a small parameter. The authors extend the method of matched asymptotic expansions initially developed for solving boundary value problems to obtain approximate solution for SPDDEs. In a series of papers [11-14], Kadalbajoo and Sharma developed uniformly convergent numerical methods using fitted mesh FDMs techniques. Swamy et al. [15-17] considered the problem and developed a numerical scheme using fitted operator finite difference techniques. Melesse et al. [18] applied initial value technique for treating the considered SPDDEs. Ranjan and Prasad [19] used modified fitted operator FDM for solving the problem. Sirisha et al. [20] developed fitted operator finite difference scheme using the procedure of domain decomposition. A number of authors have developed numerical scheme using exponentially fitted method for solving SPDDEs. To the authors' knowledge, none of them show the uniform convergence of their schemes. This motivates to treat the considered SPDDEs and formulate the uniform convergence analysis of the scheme. Our contribution in this paper is to develop higher-order uniformly convergent numerical scheme using exponentially fitted FDM and to analyse the uniform convergence of the proposed scheme.

Notation 1. The symbol $C$ is used to denote positive constant independent of $\varepsilon$ and $N$. The norm $\|$.$\| denotes the$ maximum norm.

\section{Statement of the Problem}

A class of second-order singularly perturbed differential difference equations having mixed shift on reaction terms is given by

$$
-\varepsilon u^{\prime \prime}(x)+a(x) u^{\prime}(x)+\alpha(x) u(x-\delta)+\omega(x) u(x)+\beta(x) u(x+\eta)=f(x), \quad x \in \Omega=(0,1)
$$

with the interval conditions

$$
\begin{aligned}
& u(x)=\phi(x), \quad x \in[-\delta, 0], \\
& u(x)=\psi(x), \quad x \in[1,1+\eta],
\end{aligned}
$$

where $\varepsilon \in(0,1]$ is singular perturbation parameter and $\delta$ and $\eta$ are delay and advance parameters satisfying $\delta, \eta<\varepsilon$. The functions $a(x), \alpha(x), \omega(x), \beta(x), f(x), \phi(x)$, and $\psi(x)$ are assumed to be sufficiently smooth, bounded, and independent of $\varepsilon$ for guaranteeing the existence of unique solution. The coefficient functions $\alpha(x), \omega(x)$, and $\beta(x)$ are assumed to satisfy

$$
\alpha(x)+\omega(x)+\beta(x) \geq q^{*}>0, \quad \forall x \in \bar{\Omega},
$$

for some constant $q^{*}$.

For the case where $\delta, \eta<\varepsilon$, using Taylor's series approximation for the terms with shift is appropriate [21]. So, we approximate $u(x-\delta)$ and $u(x+\eta)$ as

$$
\begin{aligned}
& u(x-\delta) \approx u(x)-\delta u^{\prime}(x)+\frac{\delta^{2}}{2} u^{\prime \prime}(x)+O\left(\delta^{3}\right), \\
& u(x+\eta) \approx u(x)+\eta u^{\prime}(x)+\frac{\eta^{2}}{2} u^{\prime \prime}(x)+O\left(\eta^{3}\right) .
\end{aligned}
$$

Using the approximation in (4) into (1)-(2) gives

$$
-c_{\varepsilon} u^{\prime \prime}(x)+p(x) u^{\prime}(x)+q(x) u(x)=f(x), \quad x \in(0,1),
$$

with the boundary conditions

$$
\begin{aligned}
& u(0)=\phi(0), \\
& u(1)=\psi(1),
\end{aligned}
$$

where $c_{\varepsilon}=\varepsilon^{2}-\left(\delta^{2} / 2\right) \alpha-\left(\eta^{2} / 2\right) \beta, \quad p(x)=a(x)-\delta \alpha(x)+$ $\eta \beta(x)$, and $q(x)=\alpha(x)+\beta(x)+\omega(x)$ for $\alpha$ and $\beta$ are lower bounds of $\alpha(x)$ and $\beta(x)$, respectively. For small values of $\delta, \eta$, equations (1)-(2) and (5)-(6) are asymptotically equivalent, since the truncated term in (5)-(6) is order of $O\left(\delta^{3}, \eta^{3}\right)$.

Let us denote differential operator $L$ for the differential equation in (5) as

$$
L u(x)=-c_{\varepsilon} u^{\prime \prime}(x)+p(x) u^{\prime}(x)+q(x) u(x) .
$$

The solution of the problem in (5)-(6) exhibits regular boundary layer of thickness $O\left(c_{\varepsilon}\right)$ and the position of the boundary layer depends on the sign of $p(x)$. If $p(x)<0$, left boundary layer exists and, for $p(x)>0$, right boundary layer exists. If $p(x), x \in[0,1]$ changes sign interior layer will occur [22].

Setting $c_{\varepsilon}=0$ in equations (5)-(6) gives the reduced problem. For the case where $p(x)>0$, it is given by

$$
\left\{\begin{array}{l}
p(x) u_{0}^{\prime}(x)+q(x) u_{0}(x)=f(x), \quad \forall x \in \Omega, \\
u_{0}(0)=\phi(0)
\end{array}\right.
$$

and, for the case where $p(x)<0$, it is given by

$$
\left\{\begin{array}{l}
p(x) u_{0}^{\prime}(x)+q(x) u_{0}(x)=f(x), \quad \forall x \in \Omega, \\
u_{0}(1)=\psi(1)
\end{array}\right.
$$

It is a first-order initial value problem; for small values of $c_{\varepsilon}$, the solution of (5)-(6) is very close to the solution of (8) or (9).

\subsection{Properties of the Analytical Solution}

Lemma 1 (The maximum principle). Let $z$ be a sufficiently smooth function defined on $\Omega$, which satisfies $z(0) \geq 0$ and $z(1) \geq 0$. Then $L z(x)>0, \forall x \in \Omega$, implies that $z(x) \geq 0, \forall x \in \bar{\Omega}$. 
Proof. Let $x^{*} \in \bar{\Omega}$ be such that $z\left(x^{*}\right)=\min _{(x) \in \bar{\Omega}} z(x)$ and suppose that $z\left(x^{*}\right)<0$. It is clear that $x^{*} \notin\{0,1\}$. Since $z\left(x^{*}\right)=\min _{(x) \in \bar{\Omega}} z(x)$ form extrema values in calculus we have $z^{\prime}\left(x^{*}\right)=0$ and $z^{\prime \prime}\left(x^{*}\right) \geq 0$ giving that $L z\left(x^{*}\right)<0$, which is contradiction to the assumption made above: $L z\left(x^{*}\right)>0, \forall x \in \Omega$. Therefore, $z(x) \geq 0, \forall x \in \Omega$.

Lemma 2 (Stability estimate). Let $u$ be the solution of (5)-(6); then, it satisfies the bound

$$
|u(x)| \leq \frac{|f|}{q^{*}}+\max \{|\phi(0)|,|\psi(1)|\},
$$

Proof. Let us define barrier functions $\vartheta_{ \pm}(x)$ as $\vartheta_{ \pm}(x)=\left(\|f\| / q^{*}\right)+\max \{\phi(0), \psi(1)\} \pm u(x)$ and apply the maximum principle to obtain the required bound.

On the boundary points, we have

$$
\begin{aligned}
& \vartheta_{ \pm}(0)=\frac{\|f\|}{q^{*}}+\max \{\phi(0), \psi(1)\} \pm u(0) \geq 0, \\
& \vartheta_{ \pm}(1)=\frac{\|f\|}{q^{*}}+\max \{\phi(0), \psi(1)\} \pm u(1) \geq 0 .
\end{aligned}
$$

On the differential operator,

where $q^{*}$ is lower bound of $q(x)$.

$$
\begin{aligned}
L \vartheta_{ \pm}(x) & =-c_{\varepsilon} \vartheta_{ \pm}^{\prime \prime}(x)+p(x) \vartheta_{ \pm}^{\prime}(x)+q(x) \vartheta_{ \pm}(x) \\
& =-c_{\varepsilon}\left(0 \pm u^{\prime \prime}(x)\right)+p(x)\left(0 \pm u^{\prime}(x)\right)+q(x)\left(\frac{\|f\|}{q^{*}}+\max \{\phi(0), \psi(1)\} \pm u(x)\right) \\
& =q(x)\left(\frac{\|f\|}{q^{*}}+\max \{\phi(0), \psi(1)\}\right) \pm f(x) \\
& \geq 0, \quad \text { since } q(x) \geq q^{*}>0,
\end{aligned}
$$

which implies that $L \vartheta_{ \pm}(x) \geq 0$. Hence, using the maximum principle in Lemma 1, we obtain $\vartheta_{ \pm}(x) \geq 0, \forall x \in \bar{\Omega}$.

Lemma 3. The derivatives of the solutions of (5)-(6) satisfy the bound

$$
\left|u^{(k)}(x)\right| \leq C\left(1+c_{\varepsilon}^{-k} \exp \left(-\frac{p^{*} x}{c_{\varepsilon}}\right)\right), \quad x \in \bar{\Omega}, 0 \leq k \leq 4,
$$

for left boundary layer problems and

$$
\left|u^{(k)}(x)\right| \leq C\left(1+c_{\varepsilon}^{-k} \exp \left(-\frac{p^{*}(1-x)}{c_{\varepsilon}}\right)\right), \quad x \in \bar{\Omega}, 0 \leq k \leq 4,
$$

for right boundary layer problems.

Proof. See [23].

\section{Numerical Scheme}

First, let us discretize the domain $\bar{\Omega}=[0,1]$ into $N$ equal number of subintervals with mesh length $h=(1 / N)$ as $\bar{\Omega}^{N}=\left\{x_{i}=i h\right\}_{0}^{N}$. Let $u(x)$ be smooth function on the domain $\bar{\Omega}=[0,1]$; then, using Taylor series approximation, we have

$$
\begin{aligned}
& u\left(x_{i+1}\right)=u_{i+1}=u_{i}+h u_{i}^{\prime}+\frac{h^{2}}{2 !} u_{i}^{\prime \prime}+\frac{h^{3}}{3 !} u_{i}^{(3)}+\frac{h^{4}}{4 !} u_{i}^{(4)}+\frac{h^{5}}{5 !} u_{i}^{(5)}+\frac{h^{6}}{6 !} u_{i}^{(6)}+O\left(h^{7}\right) \\
& u\left(x_{i-1}\right)=u_{i-1}=u_{i}-h u_{i}^{\prime}+\frac{h^{2}}{2 !} u_{i}^{\prime \prime}-\frac{h^{3}}{3 !} u_{i}^{(3)}+\frac{h^{4}}{4 !} u_{i}^{(4)}-\frac{h^{5}}{5 !} u_{i}^{(5)}+\frac{h^{6}}{6 !} u_{i}^{(6)}+O\left(h^{7}\right) .
\end{aligned}
$$

Taking the difference in (15), we obtain

$$
u_{i-1}-2 u_{i}+u_{i+1}=\frac{2 h^{2}}{2 !} u_{i}^{\prime \prime}+\frac{2 h^{4}}{4 !} u_{i}^{(4)}+\frac{2 h^{6}}{6 !} u_{i}^{(6)}+O\left(h^{8}\right) \text {. }
$$

Differentiating (16) two times gives

$$
u_{i-1}^{\prime \prime}-2 u_{i}^{\prime \prime}+u_{i+1}^{\prime \prime}=\frac{2 h^{2}}{2 !} u_{i}^{(4)}+\frac{2 h^{4}}{4 !} u_{i}^{(6)}+\frac{2 h^{6}}{6 !} u_{i}^{(8)}+O\left(h^{8}\right) .
$$

Now, multiplying (17) by $-\left(h^{2} / 12\right)$ and adding with (16) to eliminate the term with $u_{i}^{(4)}$ gives 


$$
u_{i-1}-2 u_{i}+u_{i+1}=\frac{h^{2}}{12}\left[u_{i-1}^{\prime \prime}+10 u_{i}^{\prime}+u_{i+1}^{\prime \prime}\right]+\tau,
$$

where $\tau=O\left(h^{6}\right)$. Evaluating (5) at $x_{i-1}, x_{i}$, and $x_{i+1}$, respectively, we obtain

$$
\begin{aligned}
-c_{\varepsilon} u_{i-1}^{\prime \prime} & =-p\left(x_{i-1}\right) u_{i-1}^{\prime}-q\left(x_{i-1}\right) u_{i-1}+f\left(x_{i-1}\right), \\
-c_{\varepsilon} u_{i}^{\prime \prime} & =-p\left(x_{i}\right) u_{i}^{\prime}-q\left(x_{i}\right) u_{i}+f\left(x_{i}\right), \\
-c_{\varepsilon} u_{i+1}^{\prime \prime} & =-p\left(x_{i+1}\right) u_{i+1}^{\prime}-q\left(x_{i+1}\right) u_{i+1}+f\left(x_{i+1}\right) .
\end{aligned}
$$

Next, approximate the first derivative terms $u_{i-1}^{\prime}, u_{i}^{\prime}$, and $u_{i-1}^{\prime}$ in (19), using the right shifted, central, and left shifted finite difference approximations as

$$
\begin{aligned}
& u_{i-\bar{\Gamma}}^{\prime}=\frac{u_{i-1}-4 u_{i}+3 u_{i+1}}{2 h}+h u_{i}^{\prime \prime}+O\left(h^{2}\right), \\
& u_{i}^{\prime}=\frac{u_{i+1}-u_{i-1}}{2 h}+O\left(h^{2}\right), \\
& u_{i+\bar{\Gamma}}^{\prime}=\frac{-3 u_{i-1}+4 u_{i}-u_{i+1}}{2 h}-h u_{i}^{\prime \prime}+O\left(h^{2}\right) .
\end{aligned}
$$

Substituting (20) into (19) and then (19) into (18) gives

$$
\begin{aligned}
& -\left(c_{\varepsilon}-\frac{h p_{i-1}}{12}+\frac{h p_{i+1}}{12}\right)\left(\frac{U_{i-1}-2 U_{i}+U_{i+1}}{h^{2}}\right)+\frac{p_{i-1}}{24 h}\left(-3 U_{i-1}+4 U_{i}-U_{i+1}\right) \\
& +\frac{10 p_{i}}{24 h}\left(U_{i+1}-U_{i-1}\right)+\frac{p_{i+1}}{24 h}\left(U_{i-1}-4 U_{i}+3 U_{i+1}\right)+\frac{q_{i-1}}{12} U_{i-1}+\frac{10 q_{i}}{12} U_{i}+\frac{q_{i+1}}{12} U_{i+1} \\
& =\frac{1}{12}\left[f_{i-1}+10 f_{i}+f_{i+1}\right]+O\left(h^{2}\right), \quad i=1,2, \ldots, N-1,
\end{aligned}
$$

where $p_{i-1}, p_{i}$, and $p_{i+1}$ are denoted for $p\left(x_{i-1}\right), p\left(x_{i}\right)$, and $p\left(x_{i+1}\right)$, respectively. We denote $U_{i}$ for the approximate solution of $u\left(x_{i}\right)$ in the above discretization.

To get small truncation error in boundary layer region, we apply exponentially fitted operator finite difference method (FOFDM). For developing the FOFDM, we use the theory developed in asymptotic method for treating singularly perturbed BVPs. Let us consider and treat the left and the right boundary layer cases separately.

3.1. Left Boundary Layer Problems. In this case, the boundary layer occurs near $x=0$. From the theory of singular perturbation given in [24], the zeroth-order asymptotic solution of (5)-(6) is given by

$$
u(x)=u_{0}(x)+\frac{p(0)}{p(x)}\left(\phi(0)-u_{0}(0)\right) \exp \left(-\int_{0}^{x}\left(\frac{p(x)}{c_{\varepsilon}}-\frac{q(x)}{p(x)}\right) \mathrm{d} x\right)+O\left(c_{\varepsilon}\right)
$$

where $u_{0}$ is the solution of the reduced problem. Using Taylor's series approximation for $u_{0}(x), p(x)$, and $q(x)$ centred at $x_{i}=i$ up to first order and considering $c_{\varepsilon} \longrightarrow 0$, the discretized form of (22) becomes

$$
u(i h)=u_{0}(i h)+\left(\phi(0)-u_{0}(0)\right) \exp \left(-\rho p_{i}\right),
$$

where $\rho=h / c_{\varepsilon}, h=1 / N$. Similarly, we write

$$
\begin{aligned}
& u((i+1) h)=u_{0}(i h)+\left(\phi(0)-u_{0}(0)\right) \exp \left(-\rho p_{i}(i+1)\right) \\
& u((i-1) h)=u_{0}(i h)+\left(\phi(0)-u_{0}(0)\right) \exp \left(-\rho p_{i}(i-1)\right) .
\end{aligned}
$$

To handle the effect of the perturbation parameter, exponential fitting factor $\sigma_{1}(\rho)$ is multiplied on the term containing the perturbation parameter as

$$
\begin{aligned}
& -\left[\sigma_{1}(\rho) c_{\varepsilon}-\frac{h p_{i-1}}{12}+\frac{h p_{i+1}}{12}\right]\left(\frac{U_{i-1}-2 U_{i}+U_{i+1}}{h^{2}}\right)+\frac{p_{i-1}}{24 h}\left(-3 U_{i-1}+4 U_{i}-U_{i+1}\right) \\
& +\frac{10 p_{i}}{24 h}\left(U_{i+1}-U_{i-1}\right)+\frac{p_{i+1}}{24 h}\left(U_{i-1}-4 U_{i}+3 U_{i+1}\right)+\frac{q_{i-1}}{12} U_{i-1}+\frac{10 q_{i}}{12} U_{i}+\frac{q_{i+1}}{12} U_{i+1} \\
& =\frac{1}{12}\left[f_{i-1}+10 f_{i}+f_{i+1}\right], \quad i=1,2, \ldots, N-1 .
\end{aligned}
$$


Multiplying both sides of (25) by $h$ and substituting $\rho$ for $h / c_{\varepsilon}$ and taking the limit as $h \longrightarrow 0$ give

$$
\begin{aligned}
& -\lim _{h \longrightarrow 0} \frac{\sigma_{1}(\rho)}{\rho}\left(U_{i-1}-2 U_{i}+U_{i+1}\right)+\frac{p_{0}}{24} \lim _{h \longrightarrow 0}\left(-3 U_{i-1}+4 U_{i}-U_{i+1}\right)+\frac{10 p_{0}}{24} \lim _{h \rightarrow 0}\left(U_{i+1}-U_{i-1}\right) \\
& +\frac{p_{0}}{24} \lim _{h \longrightarrow 0}\left(U_{i-1}-4 U_{i}+3 U_{i+1}\right)=0 .
\end{aligned}
$$

From (23) and (24), we obtain

$$
\left\{\begin{array}{l}
\lim _{h \longrightarrow 0}\left(U_{i-1}-2 U_{i}+U_{i+1}\right)=\left(\phi(0)-u_{0}(0)\right) e^{\left(-p_{0} i \rho\right)}\left[e^{\left(p_{0} \rho\right)}-2+e^{-\left(p_{0} \rho\right)}\right] \\
\lim _{h \longrightarrow 0}\left(-3 U_{i-1}+4 U_{i}-U_{i+1}\right)=\left(\phi(0)-u_{0}(0)\right) e^{\left(-p_{0} i \rho\right)}\left[-3 e^{\left(p_{0} \rho\right)}+4-e^{-\left(p_{0} \rho\right)}\right] \\
\lim _{h \longrightarrow 0}\left(U_{i-1}-4 U_{i}+3 U_{i+1}\right)=\left(\phi(0)-u_{0}(0)\right) e^{\left(-p_{0} i \rho\right)}\left[e^{\left(p_{0} \rho\right)}-4+3 e^{-\left(p_{0} \rho\right)}\right] \\
\lim _{h \longrightarrow 0}\left(U_{i+1}-U_{i-1}\right)=\left(\phi(0)-u_{0}(0)\right) e^{\left(-p_{0} i \rho\right)}\left[e^{\left(-p_{0} \rho\right)}-e^{\left(p_{0} \rho\right)}\right] .
\end{array}\right.
$$

Substituting (27) into (26) and simplifying give

$$
\frac{\sigma_{1}(\rho)}{\rho}\left[e^{\left(p_{0} \rho\right)}-2+e^{-\left(p_{0} \rho\right)}\right]=\frac{p_{0}}{24}\left(\left[-3 e^{\left(p_{0} \rho\right)}-e^{-\left(p_{0} \rho\right)}\right]+10\left[e^{\left(-p_{0} \rho\right)}-e^{\left(p_{0} \rho\right)}\right]+\left[e^{\left(p_{0} \rho\right)}+3 e^{-\left(p_{0} \rho\right)}\right]\right)
$$

The exponential fitting factor is obtained as

$$
L_{L}^{h} U_{i}=\frac{1}{12}\left[f_{i-1}+10 f_{i}+f_{i+1}\right], \quad i=1,2, \ldots, N-1,
$$

$$
\sigma_{1}(\rho)=\frac{\rho p(0)}{2} \operatorname{coth}\left(\frac{\rho p(0)}{2}\right) .
$$

where

Hence, the required finite difference scheme becomes

$$
\begin{aligned}
L_{L}^{h} U_{i}= & -\left[\sigma_{1}(\rho) c_{\varepsilon}-\frac{h p_{i-1}}{12}+\frac{h p_{i+1}}{12}\right]\left(\frac{U_{i-1}-2 U_{i}+U_{i+1}}{h^{2}}\right)+\frac{p_{i-1}}{24 h}\left(-3 U_{i-1}+4 U_{i}-U_{i+1}\right) \\
& +\frac{10 p_{i}}{24 h}\left(U_{i+1}-U_{i-1}\right)+\frac{p_{i+1}}{24 h}\left(U_{i-1}-4 U_{i}+3 U_{i+1}\right)+\frac{q_{i-1}}{12} U_{i-1}+\frac{10 q_{i}}{12} U_{i}+\frac{q_{i+1}}{12} U_{i+1}
\end{aligned}
$$


with the boundary values $U_{0}=\phi(0)$ and $U_{N}=\psi(1)$.
3.2. Right Boundary Layer Problems. In this case, the boundary layer occurs near $x=1$. From [24], the zerothorder asymptotic solution of (5)-(6) is given by

$$
u(x)=u_{0}(x)+\frac{p(1)}{p(x)}\left(\psi(1)-u_{0}(1)\right) \exp \left(-\int_{x}^{1}\left(\frac{p(x)}{c_{\varepsilon}}-\frac{q(x)}{p(x)}\right) \mathrm{d} x\right)+O\left(c_{\varepsilon}\right)
$$

where $u_{0}$ is the solution of the reduced problem.

Using Taylor's series approximation for $u_{0}(x), p(x)$, and $q(x)$ centred at $x_{i}=i h$ up to first order and considering $c_{\varepsilon} \longrightarrow 0$, the discretized form of (24) becomes

$$
u(i h)=u_{0}(i h)+\left(\phi(0)-u_{0}(0)\right) \exp \left(-p_{i}\left(\frac{1}{c_{\varepsilon}-\rho}\right)\right) .
$$

$$
\sigma_{2}(\rho)=\frac{\rho p(1)}{2} \operatorname{coth}\left(\frac{\rho p(1)}{2}\right) .
$$

Hence, the required finite difference scheme becomes

$$
L_{R}^{h} U_{i}=\frac{1}{12}\left[f_{i-1}+10 f_{i}+f_{i+1}\right], \quad i=1,2, \ldots, N-1,
$$

where

Using similar procedures as the left boundary layer case, the exponential fitting factor is obtained as

$$
\begin{aligned}
L_{R}^{h} U_{i}= & -\left[\sigma_{2}(\rho) c_{\varepsilon}-\frac{h p_{i-1}}{12}+\frac{h p_{i+1}}{12}\right]\left(\frac{U_{i-1}-2 U_{i}+U_{i+1}}{h^{2}}\right)+\frac{p_{i-1}}{24 h}\left(-3 U_{i-1}+4 U_{i}-U_{i+1}\right) \\
& +\frac{10 p_{i}}{24 h}\left(U_{i+1}-U_{i-1}\right)+\frac{p_{i+1}}{24 h}\left(U_{i-1}-4 U_{i}+3 U_{i+1}\right)+\frac{q_{i-1}}{12} U_{i-1}+\frac{10 q_{i}}{12} U_{i}+\frac{q_{i+1}}{12} U_{i+1} .
\end{aligned}
$$

3.3. Convergence Analysis. In this section, we show the stability and convergence analysis for the right boundary layer problems. In similar manner, it is proved for the left boundary layer case. First, we need to prove the discrete comparison principle for the scheme in (35) for guaranteeing existence of unique discrete solution.

Lemma 4 (Discrete comparison principle). Assume that, for mesh function $U_{i}$ there exists a comparison function $V_{i}$ such that $L_{R}^{h} U_{i} \leq L_{R}^{h} V_{i}, i=1,2, \ldots, N-1$ and if $U_{0} \leq V_{0}$ and $U_{N} \leq V_{N}$, then $U_{i} \leq V_{i}, \forall i, i=0,1,2, \ldots, N$.

Proof. The matrix associated with operator $L_{R}^{h}$ is of size $(N+1) \times(N+1)$ and satisfies the property of $M$-matrix. See the detailed proof in [23].

This lemma gives guarantee for the existence of unique discrete solution. In the next lemma, we discuss the uniform stability of the discrete solution.
Lemma 5 (Discrete stability estimate). The solution $U_{i}$ of the discrete scheme in (35) satisfies the following bound:

$$
\left|U_{i}\right| \leq \frac{\left\|L_{R}^{h} U_{i}\right\|}{q^{*}}+\max \left\{\left|U_{0}\right|,\left|U_{N}\right|\right\}
$$

Proof. Let $Q=\left(\left\|L_{R}^{h} U_{i}\right\| / q^{*}\right)+\max \left\{U_{0}, U_{N}\right\}$ and define barrier functions $\vartheta_{i}^{ \pm}$by $\vartheta_{i}^{ \pm}=Q \pm U_{i}$.

On the boundary points, we obtain

$$
\begin{aligned}
& \vartheta_{0}^{ \pm}=Q \pm U_{0}=\frac{\left\|L_{R}^{h} U_{i}\right\|}{q^{*}}+\max \left\{U_{0}, U_{N}\right\} \pm \phi(0) \geq 0, \\
& \vartheta_{N}^{ \pm}=Q \pm U_{N}=\frac{\left\|L_{R}^{h} U_{i}\right\|}{q^{*}}+\max \left\{U_{0}, U_{N}\right\} \pm \psi(1) \geq 0 .
\end{aligned}
$$


On the discretized spatial domain $x_{i}, 1<i<N-1$, we obtain

$$
\begin{aligned}
L_{R}^{h} \vartheta_{i}^{ \pm}= & -\left(c_{\varepsilon} \sigma(\rho)-\frac{h p_{i-1}}{12}+\frac{h p_{i+1}}{12}\right)\left(\frac{Q \pm U_{i+1}-2\left(Q \pm U_{i}\right)+Q \pm U_{i-1}}{h^{2}}\right) \\
& +\frac{p_{i-1}}{24 h}\left(-3\left(Q+U_{i-1}\right)+4\left(Q+U_{i}\right)-\left(Q+U_{i+1}\right)\right)+\frac{10 p_{i}}{24 h}\left(\left(Q+U_{i+1}\right)-\left(Q+U_{i-1}\right)\right) \\
& +\frac{p_{i+1}}{24 h}\left(\left(Q+U_{i-1}\right)-4\left(Q+U_{i}\right)+3\left(Q+U_{i+1}\right)\right) \\
& +\frac{q_{i-1}}{12}\left(Q+U_{i-1}\right)+\frac{10 q_{i}}{12}\left(Q+U_{i}\right)+\frac{q_{i+1}}{12}\left(Q+U_{i+1}\right) \\
= & \mp\left(c_{\varepsilon} \sigma(\rho)-\frac{h p_{i-1}}{12}+\frac{h p_{i+1}}{12}\right)\left(\frac{U_{i+1}-2 U_{i}+U_{i-1}}{h^{2}}\right) \pm \frac{p_{i-1}}{24 h}\left(-3 U_{i-1}+4 U_{i}-U_{i+1}\right) \\
& \pm \frac{10 p_{i}}{24 h}\left(U_{i+1}-U_{i-1}\right) \pm \frac{p_{i+1}}{24 h}\left(U_{i-1}-4 U_{i}+3 U_{i+1}\right) \pm \frac{q_{i-1}}{12} U_{i-1} \pm \frac{10 q_{i}}{12} U_{i} \pm \frac{q_{i+1}}{12} U_{i+1} \\
& +\left(\frac{q_{i-1}}{12}+\frac{10 q_{i}}{12}+\frac{q_{i+1}}{12}\right) Q \\
= & \left(\frac{q_{i-1}}{12}+\frac{10 q_{i}}{12}+\frac{q_{i+1}}{12}\right)\left(\frac{\left\|L_{R}^{h} U_{i}\right\|}{q^{*}}+\max \left\{U_{0}, U_{N}\right\}\right) \pm \frac{1}{12}\left[f_{i-1}+10 f_{i}+f_{i+1}\right] \\
& \geq 0, \quad \text { since } q\left(x_{i}\right) \geq q^{*}>0 .
\end{aligned}
$$

By the discrete comparison principle in Lemma 4, we obtain $\vartheta_{i}^{ \pm} \geq 0, \forall x_{i} \in \bar{\Omega}^{N}$. Hence, the required bound is satisfied.

Now, let us denote the right shifted, centred, and left shifted finite differences, respectively, as

$$
\begin{aligned}
& \Delta_{R} u\left(x_{i}\right)=\frac{u_{i-1}-4 u_{i}+3 u_{i+1}}{2 h}+h u_{i}^{\prime \prime}, \\
& \Delta_{C} u\left(x_{i}\right)=\frac{u_{i+1}-u_{i-1}}{2 h}, \\
& \Delta_{L} u\left(x_{i}\right)=\frac{-3 u_{i-1}+4 u_{i}-u_{i+1}}{2 h}-h u_{i}^{\prime \prime} .
\end{aligned}
$$
bound

Using Taylor's series approximation, we obtain the

$$
\begin{aligned}
\left\|u_{i-1}^{\prime}-\Delta_{L} u\left(x_{i}\right)\right\| & \leq C N^{-2}\left\|u^{(4)}\left(x_{i}\right)\right\|, \\
\left\|u_{i}^{\prime}-\Delta_{C} u\left(x_{i}\right)\right\| & \leq C N^{-2}\left\|u^{\prime \prime}\left(x_{i}\right)\right\|, \\
\left\|u_{i+1}^{\prime}-\Delta_{R} u\left(x_{i}\right)\right\| & \leq C N^{-2}\left\|u^{(4)}\left(x_{i}\right)\right\|,
\end{aligned}
$$

where $\left\|u^{(k)}\left(x_{i}\right)\right\|=\max _{x_{0} \leq x_{i} \leq x_{N}}\left|u^{(k)}\left(x_{i}\right)\right|, k=2,4$. Similarly, we have

$$
\begin{aligned}
\left\|u^{\prime \prime}\left(x_{i}\right)-D^{+} D^{-} u\left(x_{i}\right)\right\| & \leq C N^{-2}\left\|u^{(4)}\left(x_{i}\right)\right\|, \\
\left\|D^{+} D^{-} u\left(x_{i}\right)\right\| & \leq C\left\|u^{\prime \prime}\left(x_{i}\right)\right\| .
\end{aligned}
$$

Now, for $\rho>0, C_{1}$ and $C_{2}$ are constants, and we have $|\rho \operatorname{coth}(\rho)-1| \leq C_{1} \rho^{2}$, for $\rho \leq 1$. For $\rho \longrightarrow \infty$, since $\lim _{\rho \longrightarrow \infty} \operatorname{coth}(\rho)=1,|\rho \operatorname{coth}(\rho)-1| \leq C_{1} \rho$ is given. In general, for all $\rho>0$, we write

$$
C_{1} \frac{\rho^{2}}{\rho+1} \leq \rho \operatorname{coth}(\rho)-1 \leq C_{2} \frac{\rho^{2}}{\rho+1}
$$

implying that

$$
c_{\varepsilon}\left[p\left(x_{i}\right) \frac{\rho}{2} \operatorname{coth}\left(p(1) \frac{\rho}{2}\right)-1\right] \leq c_{\varepsilon} \frac{\left(N^{-1} / c_{\varepsilon}\right)^{2}}{\left(N^{-1} / c_{\varepsilon}\right)+1}=\frac{N^{-2}}{N^{-1}+c_{\varepsilon}} .
$$

The following theorem gives truncation error bound of the proposed scheme.

Theorem 1. Let $u\left(x_{i}\right)$ and $U_{i}$ be solutions of (5)-(6) and (35), respectively. Then the following error estimate holds: 
$\left\|L_{R}^{h}\left(u\left(x_{i}\right)-U_{i}\right)\right\| \leq \frac{C N^{-2}}{N^{-1}+c_{\varepsilon}}\left(1+c_{\varepsilon}^{-4} \max _{x_{1} \leq x_{i} \leq x_{N-1}} \exp \left(-\frac{p^{*}\left(1-x_{i}\right)}{c_{\varepsilon}}\right)\right)$.

Proof. Consider the truncation error that is given by

$$
\begin{aligned}
\left\|L^{h} u\left(x_{i}\right)-L_{R}^{h} U_{i}\right\| \leq & \left\|-c_{\varepsilon}\left(u^{\prime \prime}\left(x_{i}\right)-\sigma(\rho) D^{+} D^{-} u\left(x_{i}\right)\right)\right\|+\left\|\frac{p_{i-1}}{12}\left(u_{i-1}^{\prime}-\Delta_{L} u\left(x_{i}\right)\right)\right\| \\
& +\left\|\frac{10 p_{i}}{12}\left(u_{i}^{\prime}-\Delta_{C} u\left(x_{i}\right)\right)\right\|+\left\|\frac{p_{i+1}}{12}\left(u_{i+1}^{\prime}-\Delta_{R} u\left(x_{i}\right)\right)\right\| \\
\leq & \left\|-c_{\varepsilon}\left[p(1) \frac{\rho}{2} \cot h\left(p(1) \frac{\rho}{2}\right)-1\right] D^{+} D^{-} u\left(x_{i}\right)\right\|+\left\|c_{\varepsilon}\left(u^{\prime \prime}\left(x_{i}\right)-D^{+} D^{-} u\left(x_{i}\right)\right)\right\| \\
& +\left\|\frac{p_{i-1}}{12}\left(u_{i-1}^{\prime}-\Delta_{L} u\left(x_{i}\right)\right)\right\|+\left\|\frac{10 p_{i}}{12}\left(u_{i}^{\prime}-\Delta_{C} u\left(x_{i}\right)\right)\right\|+\left\|\frac{p_{i+1}}{12}\left(u_{i+1}^{\prime}-\Delta_{R} u\left(x_{i}\right)\right)\right\| .
\end{aligned}
$$

Using the bounds in (44), (41), and (42) gives

$$
\begin{aligned}
\left\|L^{h}\left(u\left(x_{i}\right)-U_{i}\right)\right\| & \leq \frac{C N^{-2}}{N^{-1}+c_{\varepsilon}}\left\|u^{\prime \prime}\left(x_{i}\right)\right\|+c_{\varepsilon} C N^{-2}\left\|u^{(4)}\left(x_{i}\right)\right\|+C N^{-2}\left\|u^{(4)}\left(x_{i}\right)\right\| \\
& \leq \frac{C N^{-2}}{N^{-1}+c_{\varepsilon}}\left\|u^{\prime \prime}\left(x_{i}\right)\right\|+C N^{-2}\left\|u^{(4)}\left(x_{i}\right)\right\| .
\end{aligned}
$$

Substituting the bounds for the derivatives of the solution in Lemma 3, we obtain

$$
\begin{aligned}
\left\|L^{h}\left(u\left(x_{i}\right)-U_{i}\right)\right\| \leq & \frac{C N^{-2}}{N^{-1}+c_{\varepsilon}}\left(1+c_{\varepsilon}^{-2} \exp \left(-\frac{p^{*}\left(1-x_{i}\right)}{c_{\varepsilon}}\right)\right) \\
& +C N^{-2}\left(1+c_{\varepsilon}^{-4} \exp \left(-\frac{p^{*}\left(1-x_{i}\right)}{c_{\varepsilon}}\right)\right) .
\end{aligned}
$$

Since $c_{\varepsilon}^{-2} \leq c_{\varepsilon}^{-4}$, we obtain

$$
\begin{aligned}
\left\|L^{h}\left(u\left(x_{i}\right)-U_{i}\right)\right\| & \leq \frac{C N^{-2}}{N^{-1}+c_{\varepsilon}} \\
& \cdot\left(1+c_{\varepsilon}^{-4} \max _{x_{1} \leq x_{i} \leq x_{N-1}} \exp \left(-\frac{p^{*}\left(1-x_{i}\right)}{c_{\varepsilon}}\right)\right) .
\end{aligned}
$$

Lemma 6. For $c_{\varepsilon} \longrightarrow 0$, and for given fixed $N$, we obtain

$$
\begin{aligned}
& \lim _{c_{\varepsilon} \longrightarrow 0} \max _{x_{j}} \frac{\exp \left(-\left(p^{*} x_{j} / c_{\varepsilon}\right)\right)}{c_{\varepsilon}^{m}}=0, \\
& \lim _{c_{\varepsilon} \longrightarrow 0} \max _{x_{j}} \frac{\exp \left(-\left(p^{*} x_{j} / c_{\varepsilon}\right)\right)}{c_{\varepsilon}^{m}}=0, \quad m=1,2,3, \ldots,
\end{aligned}
$$

where $x_{j}=j h, h=1 / N, \forall j=1,2, \ldots, N-1$.

Proof. See [25-27].

Theorem 2. Under the hypothesis of boundedness of discrete solution, the solution of the discrete schemes in (30) satisfies the following uniform error bound:

$$
\sup _{0<c_{\varepsilon} \ll 1}\left\|u\left(x_{i}\right)-U_{i}\right\| \leq C N^{-1} \text {. }
$$

Proof. Substituting the results in Lemma 6 into Theorem 1, applying Lemma 5 gives the required bound.

3.4. Richardson Extrapolation. We apply the Richardson extrapolation technique to accelerate the rate of convergence of the proposed scheme. Richardson extrapolation is a convergence acceleration technique that involves combination of two computed approximations of solution. Interested reader can see the details of Richardson extrapolation in [28]. From (34) and Lemma 6, we obtain

$$
\left|L_{R}^{h}\left(u\left(x_{i}\right)-U_{i}\right)\right| \leq C\left(N^{-1}+N^{-2}\right),
$$

where $u\left(x_{i}\right)$ and $U_{i}$ are the exact and approximate solutions of (5)-(6), respectively. Applying Lemma 5 in (52) gives

$$
\left|u\left(x_{i}\right)-U_{i}\right| \leq C N^{-1}+O\left(N^{-2}\right) \text {. }
$$


Let us denote $U_{i}^{2 N}$ for an approximate solution on $2 \mathrm{~N}$ number of mesh points by including the mid-points. Applying the same procedure as in (52) and (53) on $2 N$ number of mesh points, we obtain

$$
\left|u\left(x_{i}\right)-U_{i}^{2 N}\right| \leq C(2 N)^{-1}+O\left(N^{-2}\right) .
$$

Combining (53) and (54) for removing the term $\mathrm{CN}^{-1}$ results in

$$
\left|u\left(x_{i}\right)-\left(2 U_{i}^{2 N}-U_{i}\right)\right| \leq C N^{-2},
$$

giving

$$
U_{i}^{\mathrm{ext}}=2 U_{i}^{2 N}-U_{i},
$$

as the extrapolated solution. The error bound for the extrapolated solution in (56) becomes

$$
\sup _{0<c_{\varepsilon} \ll 1}\left\|u\left(x_{i}\right)-U_{i}^{\mathrm{ext}}\right\| \leq C N^{-2} .
$$

\section{Examples and Numerical Results}

In this section, we consider numerical examples to illustrate the theoretical findings of the developed schemes.

Example 1. Consider the problem

$$
-\varepsilon u^{\prime \prime}(x)+u^{\prime}(x)+2 u(x-\delta)+5 u(x)-u(x+\eta)=0,
$$

with interval conditions $u(x)=1,-\delta \leq x \leq 0, u(x)=$ $-1,1 \leq x \leq 1+\eta$.

Example 2. Consider the problem

$$
\begin{aligned}
& -\varepsilon u^{\prime \prime}(x)+\left(1+\exp \left(x^{2}\right)\right) u^{\prime}(x)+x u(x-\delta) \\
& -x^{2} u(x)+(1-\exp (-x)) u(x+\eta)=-1,
\end{aligned}
$$

with interval conditions $u(x)=1,-\delta \leq x \leq 0, u(x)=$ $-1,1 \leq x \leq 1+\eta$.

Example 3. Consider the problem

$$
-\varepsilon u^{\prime \prime}(x)-0.5 u^{\prime}(x)+3 u(x-\delta)+2 u(x)-2 u(x+\eta)=-1,
$$

with interval conditions $u(x)=1,-\delta \leq x \leq 0, u(x)=$ $0,1 \leq x \leq 1+\eta$.

Example 4. Consider the problem

$$
\varepsilon u^{\prime \prime}(x)+2.5 u^{\prime}(x)-2 \exp (x) u(x-\delta)-u(x)-x u(x+\eta)=1,
$$

with interval conditions $u(x)=1,-\delta \leq x \leq 0, u(x)=$ $1,1 \leq x \leq 1+\eta$.

The exact solution of the constant coefficient interval value problem

$$
\varepsilon u^{\prime \prime}(x)+a u^{\prime}(x)+\alpha u(x-\delta)+\omega u(x)+\beta u(x+\eta)=f(x),
$$

on $\Omega=(0,1)$, with the interval conditions $u(x)=\phi(x), x \in[-\delta, 0], u(x)=\psi(x), x \in[1,1+\eta], \quad$ is given by

$$
u(x)=c_{1} e^{m_{1} x}+c_{2} e^{m_{2} x}+\frac{f}{c},
$$

where $c=\alpha+\omega+\beta, \quad c_{1}=\left(-f+\psi(1) c+e^{m_{2}}(f-\phi(0) c)\right) /$ $c\left(e^{m_{1}}-e^{m_{2}}\right), \quad c_{2}=\left(-f+\psi(1) c+e^{m_{1}}(f-\phi(0) c)\right) / c\left(e^{m_{1}}\right.$ $\left.-e^{m_{2}}\right), \quad m_{1}=\left(-(a-\delta \alpha+\eta \beta)+\sqrt{(a-\delta \alpha+\eta \beta)^{2}-4 c \varepsilon}\right) / 2 \varepsilon$, and $m_{2}=\left(-(a-\delta \alpha+\eta \beta)-\sqrt{(a-\delta \alpha+\eta \beta)^{2}-4 c \varepsilon}\right) / 2 \varepsilon$.

The exact solutions of the variable coefficient problems are not known. So, we use the procedure of the double mesh technique to calculate maximum absolute error. The maximum absolute error is defined as

$$
E_{\varepsilon}^{N}=\max _{0 \leq i \leq N}\left|U_{i}^{N}-U_{i}^{2 N}\right|,
$$

where $U_{i}^{N}$ denotes the solution of the problem on $N$ number of mesh points and $U_{i}^{2 N}$ denotes the numerical solution on $2 N$ number of mesh points by including the mid-points $x_{(i+1) / 2}$ into the mesh numbers. The uniform error estimate is defined as

$$
E^{N}=\max _{\varepsilon}\left|E_{\varepsilon}^{N}\right|
$$

The rate of convergence of the scheme is given by

$$
R_{\varepsilon}^{N}=\log _{2}\left(\frac{E_{\varepsilon}^{N}}{E_{\varepsilon}^{2 N}}\right)
$$

and the uniform rate of convergence is given as

$$
R^{N}=\log _{2}\left(\frac{E^{N}}{E^{2 N}}\right)
$$

In Tables 1-8, the maximum absolute error of Examples 1-4 using the proposed scheme is given. In Tables $1,3,5$, and 7, the maximum absolute error before the Richardson extrapolation is given, and in Tables 2, 4, 6, and 8 , the maximum absolute error after the Richardson extrapolation is given. As one observes in the tables, for each number of mesh interval $N$ as $\varepsilon \longrightarrow 0$, the maximum absolute error becomes stable and uniform. This indicates that the proposed scheme convergence is independent of the perturbation parameter $\varepsilon$. In the last two rows of each table, we observe the $\varepsilon$-uniform error and the $\varepsilon$-uniform rate of convergence of the scheme. The scheme before the extrapolation gives first-order uniform convergence and the extrapolated scheme gives second-order uniform convergence. In Tables 9 and 10, we compare the maximum absolute error of the proposed scheme with recently published papers in $[14,15,19]$. As one observes, the proposed scheme gives more accurate result. 
TABle 1: Maximum absolute error of Example 1 before Richardson extrapolation for $\delta=0.6 \varepsilon$ and $\eta=0.5 \varepsilon$.

\begin{tabular}{|c|c|c|c|c|c|c|}
\hline$\varepsilon \downarrow$ & $N \longrightarrow 8$ & 16 & 32 & 64 & 128 & 256 \\
\hline $2^{0}$ & $1.3808 e-03$ & $3.4045 e-04$ & $8.4818 e-05$ & $2.1193 e-05$ & $5.2970 e-06$ & $1.3242 e-06$ \\
\hline $2^{-4}$ & $1.7200 e-02$ & $5.0151 e-03$ & $1.4739 e-03$ & $3.5993 e-04$ & $9.0018 e-05$ & $2.2464 e-05$ \\
\hline $2^{-8}$ & $4.1828 e-02$ & $2.6301 e-02$ & $1.4357 e-02$ & $6.0620 e-03$ & $1.8873 e-03$ & $5.0407 e-04$ \\
\hline $2^{-12}$ & $4.1711 e-02$ & $2.6194 e-02$ & $1.4838 e-02$ & $7.9779 e-03$ & $4.1434 e-03$ & $2.1118 e-03$ \\
\hline $2^{-16}$ & $4.1704 e-02$ & $2.6186 e-02$ & $1.4834 e-02$ & $7.9750 e-03$ & $4.1418 e-03$ & $2.1124 e-03$ \\
\hline $2^{-20}$ & $4.1703 e-02$ & $2.6186 e-02$ & $1.4833 e-02$ & $7.9748 e-03$ & $4.1417 e-03$ & $2.1123 e-03$ \\
\hline $2^{-24}$ & $4.1703 e-02$ & $2.6186 e-02$ & $1.4833 e-02$ & $7.9748 e-03$ & $4.1417 e-03$ & $2.1123 e-03$ \\
\hline $2^{-28}$ & $4.1703 e-02$ & $2.6186 e-02$ & $1.4833 e-02$ & $7.9748 e-03$ & $4.1417 e-03$ & $2.1123 e-03$ \\
\hline $2^{-32}$ & $4.1703 e-02$ & $2.6186 e-02$ & $1.4833 e-02$ & $7.9748 e-03$ & $4.1417 e-03$ & $2.1123 e-03$ \\
\hline $2^{-36}$ & $4.1703 e-02$ & $2.6186 e-02$ & $1.4833 e-02$ & $7.9748 e-03$ & $4.1417 e-03$ & $2.1123 e-03$ \\
\hline $2^{-40}$ & $4.1703 e-02$ & $2.6186 e-02$ & $1.4833 e-02$ & $7.9748 e-03$ & $4.1417 e-03$ & $2.1123 e-03$ \\
\hline$E^{N}$ & $4.1703 e-02$ & $2.6186 e-02$ & $1.4833 e-02$ & $7.9748 e-03$ & $4.1417 e-03$ & $2.1123 e-03$ \\
\hline$R^{N}$ & 0.6714 & 0.8200 & 0.8953 & 0.9452 & 0.9714 & - \\
\hline
\end{tabular}

TABle 2: Maximum absolute error of Example 1 after Richardson extrapolation for $\delta=0.6 \varepsilon \eta=0.5 \varepsilon$.

\begin{tabular}{|c|c|c|c|c|c|c|}
\hline$\varepsilon \downarrow$ & $N \longrightarrow 8$ & 16 & 32 & 64 & 128 & 256 \\
\hline $2^{0}$ & $3.4045 e-04$ & $2.1193 e-05$ & $1.3242 e-06$ & $8.2744 e-08$ & $5.3296 e-09$ & $1.3016 e-09$ \\
\hline $2^{-4}$ & $5.0151 e-03$ & $3.5993 e-04$ & $2.2464 e-05$ & $1.4036 e-06$ & $8.7720 e-08$ & $2.2310 e-08$ \\
\hline $2^{-8}$ & $2.6301 e-02$ & $6.0620 e-03$ & $5.0407 e-04$ & $3.3479 e-05$ & $2.0797 e-06$ & $4.2147 e-07$ \\
\hline $2^{-12}$ & $2.6194 e-02$ & $7.9779 e-03$ & $2.1118 e-03$ & $4.0826 e-04$ & $3.2897 e-05$ & $5.2346 e-06$ \\
\hline $2^{-16}$ & $2.6186 e-02$ & $7.9750 e-03$ & $2.1124 e-03$ & $5.3615 e-04$ & $1.3446 e-04$ & $3.2541 e-05$ \\
\hline $2^{-20}$ & $2.6186 e-02$ & $7.9748 e-03$ & $2.1123 e-03$ & $5.3614 e-04$ & $1.3446 e-04$ & $3.2541 e-05$ \\
\hline $2^{-24}$ & $2.6186 e-02$ & $7.9748 e-03$ & $2.1123 e-03$ & $5.3614 e-04$ & $1.3446 e-04$ & $3.2541 e-05$ \\
\hline $2^{-28}$ & $2.6186 e-02$ & $7.9748 e-03$ & $2.1123 e-03$ & $5.3614 e-04$ & $1.3446 e-04$ & $3.2541 e-05$ \\
\hline $2^{-32}$ & $2.6186 e-02$ & $7.9748 e-03$ & $2.1123 e-03$ & $5.3614 e-04$ & $1.3446 e-04$ & $3.2541 e-05$ \\
\hline $2^{-36}$ & $2.6186 e-02$ & $7.9748 e-03$ & $2.1123 e-03$ & $5.3614 e-04$ & $1.3446 e-04$ & $3.2541 e-05$ \\
\hline $2^{-40}$ & $2.6186 e-02$ & $7.9748 e-03$ & $2.1123 e-03$ & $5.3614 e-04$ & $1.3446 e-04$ & $3.2541 e-05$ \\
\hline$E^{N}$ & $2.6186 e-02$ & $7.9748 e-03$ & $2.1123 e-03$ & $5.3614 e-04$ & $1.3446 e-04$ & $3.2541 e-05$ \\
\hline$R^{N}$ & 1.7153 & 1.9166 & 1.9781 & 1.9954 & 2.0468 & - \\
\hline
\end{tabular}

TABle 3: Maximum absolute error of Example 2 before Richardson extrapolation for $\delta=0.6 \varepsilon$ and $\eta=0.5 \varepsilon$.

\begin{tabular}{|c|c|c|c|c|c|c|}
\hline$\varepsilon \downarrow$ & $N \longrightarrow 8$ & 16 & 32 & 64 & 128 & 256 \\
\hline $2^{0}$ & $3.4492 e-03$ & $1.6925 e-03$ & $8.3747 e-04$ & $4.1601 e-04$ & $2.0724 e-04$ & $1.0343 e-04$ \\
\hline $2^{-4}$ & $2.2763 e-02$ & $7.6376 e-03$ & $1.4739 e-03$ & $1.5941 e-03$ & $7.8163 e-04$ & $3.8784 e-04$ \\
\hline $2^{-8}$ & $2.3670 e-02$ & $1.1054 e-02$ & $5.2737 e-03$ & $2.5716 e-03$ & $1.2314 e-03$ & $4.9403 e-04$ \\
\hline $2^{-12}$ & $2.3670 e-02$ & $1.1054 e-02$ & $5.2735 e-03$ & $2.5654 e-03$ & $1.2638 e-03$ & $6.2704 e-04$ \\
\hline $2^{-16}$ & $2.3670 e-02$ & $1.1054 e-02$ & $5.2735 e-03$ & $2.5654 e-03$ & $1.2638 e-03$ & $6.2704 e-04$ \\
\hline $2^{-20}$ & $2.3670 e-02$ & $1.1054 e-02$ & $5.2735 e-03$ & $2.5654 e-03$ & $1.2638 e-03$ & $6.2704 e-04$ \\
\hline $2^{-24}$ & $2.3670 e-02$ & $1.1054 e-02$ & $5.2735 e-03$ & $2.5654 e-03$ & $1.2638 e-03$ & $6.2704 e-04$ \\
\hline $2^{-28}$ & $2.3670 e-02$ & $1.1054 e-02$ & $5.2735 e-03$ & $2.5654 e-03$ & $1.2638 e-03$ & $6.2704 e-04$ \\
\hline $2^{-32}$ & $2.3670 e-02$ & $1.1054 e-02$ & $5.2735 e-03$ & $2.5654 e-03$ & $1.2638 e-03$ & $6.2704 e-04$ \\
\hline $2^{-36}$ & $2.3670 e-02$ & $1.1054 e-02$ & $5.2735 e-03$ & $2.5654 e-03$ & $1.2638 e-03$ & $6.2704 e-04$ \\
\hline $2^{-40}$ & $2.3670 e-02$ & $1.1054 e-02$ & $5.2735 e-03$ & $2.5654 e-03$ & $1.2638 e-03$ & $6.2704 e-04$ \\
\hline$E^{N}$ & $2.3670 e-02$ & $1.1054 e-02$ & $5.2735 e-03$ & $2.5654 e-03$ & $1.2638 e-03$ & $6.2704 e-04$ \\
\hline$R^{N}$ & 1.0985 & 1.0677 & 1.0396 & 1.0214 & 1.0111 & - \\
\hline
\end{tabular}


TABle 4: Maximum absolute error of Example 2 after Richardson extrapolation for $\delta=0.6 \varepsilon$ and $\eta=0.5 \varepsilon$.

\begin{tabular}{|c|c|c|c|c|c|c|}
\hline$\varepsilon \downarrow$ & $N \longrightarrow 8$ & 16 & 32 & 64 & 128 & 256 \\
\hline $2^{0}$ & $1.6925 e-03$ & $4.1601 e-04$ & $1.0343 e-04$ & $2.5819 e-05$ & $6.4524 e-06$ & $1.1321 e-06$ \\
\hline $2^{-4}$ & $7.6376 e-03$ & $1.5941 e-03$ & $3.8784 e-04$ & $9.6460 e-05$ & $2.4085 e-05$ & $7.6001 e-06$ \\
\hline $2^{-8}$ & $1.1054 e-02$ & $2.5716 e-03$ & $4.9403 e-04$ & $1.0586 e-04$ & $2.6498 e-05$ & $8.0231 e-06$ \\
\hline $2^{-12}$ & $1.1054 e-02$ & $2.5654 e-03$ & $6.2704 e-04$ & $1.5625 e-04$ & $3.0982 e-05$ & $8.2543 e-06$ \\
\hline $2^{-16}$ & $1.1054 e-02$ & $2.5654 e-03$ & $6.2704 e-04$ & $1.5584 e-04$ & $3.8901 e-05$ & $9.2541 e-06$ \\
\hline $2^{-20}$ & $1.1054 e-02$ & $2.5654 e-03$ & $6.2704 e-04$ & $1.5584 e-04$ & $3.8901 e-05$ & $9.2541 e-06$ \\
\hline $2^{-24}$ & $1.1054 e-02$ & $2.5654 e-03$ & $6.2704 e-04$ & $1.5584 e-04$ & $3.8901 e-05$ & $9.2541 e-06$ \\
\hline $2^{-28}$ & $1.1054 e-02$ & $2.5654 e-03$ & $6.2704 e-04$ & $1.5584 e-04$ & $3.8901 e-05$ & $9.2541 e-06$ \\
\hline $2^{-32}$ & $1.1054 e-02$ & $2.5654 e-03$ & $6.2704 e-04$ & $1.5584 e-04$ & $3.8901 e-05$ & $9.2541 e-06$ \\
\hline $2^{-36}$ & $1.1054 e-02$ & $2.5654 e-03$ & $6.2704 e-04$ & $1.5584 e-04$ & $3.8901 e-05$ & $9.2541 e-06$ \\
\hline $2^{-40}$ & $1.1054 e-02$ & $2.5654 e-03$ & $6.2704 e-04$ & $1.5584 e-04$ & $3.8901 e-05$ & $9.2541 e-06$ \\
\hline$E^{N}$ & $1.1054 e-02$ & $2.5654 e-03$ & $6.2704 e-04$ & $1.5584 e-04$ & $3.8901 e-05$ & $9.2541 e-06$ \\
\hline$R^{N}$ & 2.1073 & 2.0326 & 2.0085 & 2.0022 & 2.0716 & - \\
\hline
\end{tabular}

Table 5: Maximum absolute error of Example 3 before Richardson extrapolation for $\delta=0.6 \varepsilon$ and $\eta=0.5 \varepsilon$.

\begin{tabular}{|c|c|c|c|c|c|c|}
\hline$\varepsilon \downarrow$ & $N \longrightarrow 8$ & 16 & 32 & 64 & 128 & 256 \\
\hline $2^{0}$ & $1.1318 e-03$ & $2.7968 e-04$ & $6.9551 e-05$ & $1.7366 e-05$ & $4.3405 e-06$ & $1.0850 e-06$ \\
\hline $2^{-4}$ & $1.4786 e-02$ & $3.6872 e-03$ & $8.5968 e-04$ & $2.1118 e-04$ & $5.2578 e-05$ & $1.3152 e-05$ \\
\hline $2^{-8}$ & $4.7613 e-02$ & $2.9531 e-02$ & $1.4879 e-02$ & $5.5516 e-03$ & $1.4704 e-03$ & $3.3684 e-04$ \\
\hline $2^{-12}$ & $4.8375 e-02$ & $3.0553 e-02$ & $1.7528 e-02$ & $9.4597 e-03$ & $4.9243 e-03$ & $2.4698 e-03$ \\
\hline $2^{-16}$ & $4.8423 e-02$ & $3.0586 e-02$ & $1.7549 e-02$ & $9.4718 e-03$ & $4.9323 e-03$ & $2.5185 e-03$ \\
\hline $2^{-20}$ & $4.8426 e-02$ & $3.0589 e-02$ & $1.7550 e-02$ & $9.4725 e-03$ & $4.9327 e-03$ & $2.5187 e-03$ \\
\hline $2^{-24}$ & $4.8426 e-02$ & $3.0589 e-02$ & $1.7550 e-02$ & $9.4725 e-03$ & $4.9328 e-03$ & $2.5187 e-03$ \\
\hline $2^{-28}$ & $4.8426 e-02$ & $3.0589 e-02$ & $1.7550 e-02$ & $9.4725 e-03$ & $4.9328 e-03$ & $2.5187 e-03$ \\
\hline $2^{-32}$ & $4.8426 e-02$ & $3.0589 e-02$ & $1.7550 e-02$ & $9.4725 e-03$ & $4.9328 e-03$ & $2.5187 e-03$ \\
\hline $2^{-36}$ & $4.8426 e-02$ & $3.0589 e-02$ & $1.7550 e-02$ & $9.4725 e-03$ & $4.9328 e-03$ & $2.5187 e-03$ \\
\hline $2^{-40}$ & $4.8426 e-02$ & $3.0589 e-02$ & $1.7550 e-02$ & $9.4725 e-03$ & $4.9328 e-03$ & $2.5187 e-03$ \\
\hline$E^{N}$ & $4.8426 e-02$ & $3.0589 e-02$ & $1.7550 e-02$ & $9.4725 e-03$ & $4.9328 e-03$ & $2.5187 e-03$ \\
\hline$R^{N}$ & 0.6628 & 0.8015 & 0.8897 & 0.9413 & 0.9697 & - \\
\hline
\end{tabular}

TABLe 6: Maximum absolute error of Example 3 after Richardson extrapolation for $\delta=0.6 \varepsilon$ and $\eta=0.5 \varepsilon$.

\begin{tabular}{|c|c|c|c|c|c|c|}
\hline$\varepsilon \downarrow$ & $N \longrightarrow 8$ & 16 & 32 & 64 & 128 & 256 \\
\hline $2^{0}$ & $2.7968 e-04$ & $1.7366 e-05$ & $1.0850 e-06$ & $6.7812 e-08$ & $4.2386 e-09$ & $1.0101 e-09$ \\
\hline $2^{-4}$ & $3.6872 e-03$ & $2.1118 e-04$ & $1.3152 e-05$ & $8.2173 e-07$ & $5.0203 e-08$ & $1.2341 e-08$ \\
\hline $2^{-8}$ & $2.9531 e-02$ & $5.5516 e-03$ & $3.3684 e-04$ & $2.0489 e-05$ & $1.2809 e-06$ & $3.7125 e-07$ \\
\hline $2^{-12}$ & $3.0553 e-02$ & $9.4597 e-03$ & $2.4698 e-03$ & $3.8494 e-04$ & $2.2756 e-05$ & $4.8133 e-06$ \\
\hline $2^{-16}$ & $3.0586 e-02$ & $9.4718 e-03$ & $2.5185 e-03$ & $6.3984 e-04$ & $1.5770 e-04$ & $3.8042 e-05$ \\
\hline $2^{-20}$ & $3.0589 e-02$ & $9.4725 e-03$ & $2.5187 e-03$ & $6.3989 e-04$ & $1.6062 e-04$ & $3.9001 e-05$ \\
\hline $2^{-24}$ & $3.0589 e-02$ & $9.4725 e-03$ & $2.5187 e-03$ & $6.3989 e-04$ & $1.6062 e-04$ & $3.9001 e-05$ \\
\hline $2^{-28}$ & $3.0589 e-02$ & $9.4725 e-03$ & $2.5187 e-03$ & $6.3989 e-04$ & $1.6062 e-04$ & $3.9001 e-05$ \\
\hline $2^{-32}$ & $3.0589 e-02$ & $9.4725 e-03$ & $2.5187 e-03$ & $6.3989 e-04$ & $1.6062 e-04$ & $3.9001 e-05$ \\
\hline $2^{-36}$ & $3.0589 e-02$ & $9.4725 e-03$ & $2.5187 e-03$ & $6.3989 e-04$ & $1.6062 e-04$ & $3.9001 e-05$ \\
\hline $2^{-40}$ & $3.0589 e-02$ & $9.4725 e-03$ & $2.5187 e-03$ & $6.3989 e-04$ & $1.6062 e-04$ & $3.9001 e-05$ \\
\hline$E^{N}$ & $3.0589 e-02$ & $9.4725 e-03$ & $2.5187 e-03$ & $6.3989 e-04$ & $1.6062 e-04$ & $3.9001 e-05$ \\
\hline$R^{N}$ & 1.6912 & 1.9111 & 1.9768 & 1.9942 & 2.0421 & - \\
\hline
\end{tabular}


TABLe 7: Maximum absolute error of Example 4 before Richardson extrapolation for $\delta=0.6 \varepsilon$ and $\eta=0.5 \varepsilon$.

\begin{tabular}{|c|c|c|c|c|c|c|}
\hline$\varepsilon \downarrow$ & $N \longrightarrow 8$ & 16 & 32 & 64 & 128 & 256 \\
\hline $2^{0}$ & $9.0320 e-03$ & $4.5788 e-03$ & $2.2918 e-03$ & $1.1469 e-03$ & $5.7338 e-04$ & $2.8666 e-04$ \\
\hline $2^{-4}$ & $3.7979 e-02$ & $1.6150 e-02$ & $6.1639 e-03$ & $2.4525 e-03$ & $1.0562 e-03$ & $4.8456 e-04$ \\
\hline $2^{-8}$ & $4.3631 e-02$ & $2.4943 e-02$ & $1.3421 e-02$ & $6.9160 e-03$ & $3.1649 e-03$ & $1.2055 e-03$ \\
\hline $2^{-12}$ & $4.3723 e-02$ & $2.4996 e-02$ & $1.3451 e-02$ & $7.0011 e-03$ & $3.5749 e-03$ & $1.8068 e-03$ \\
\hline $2^{-16}$ & $4.3728 e-02$ & $2.4999 e-02$ & $1.3452 e-02$ & $7.0020 e-03$ & $3.5755 e-03$ & $1.8071 e-03$ \\
\hline $2^{-20}$ & $4.3729 e-02$ & $2.4999 e-02$ & $1.3452 e-02$ & $7.0021 e-03$ & $3.5755 e-03$ & $1.8071 e-03$ \\
\hline $2^{-24}$ & $4.3729 e-02$ & $2.4999 e-02$ & $1.3452 e-02$ & $7.0021 e-03$ & $3.5755 e-03$ & $1.8071 e-03$ \\
\hline $2^{-28}$ & $4.3729 e-02$ & $2.4999 e-02$ & $1.3452 e-02$ & $7.0021 e-03$ & $3.5755 e-03$ & $1.8071 e-03$ \\
\hline $2^{-32}$ & $4.3729 e-02$ & $2.4999 e-02$ & $1.3452 e-02$ & $7.0021 e-03$ & $3.5755 e-03$ & $1.8071 e-03$ \\
\hline $2^{-36}$ & $4.3729 e-02$ & $2.4999 e-02$ & $1.3452 e-02$ & $7.0021 e-03$ & $3.5755 e-03$ & $1.8071 e-03$ \\
\hline $2^{-40}$ & $4.3729 e-02$ & $2.4999 e-02$ & $1.3452 e-02$ & $7.0021 e-03$ & $3.5755 e-03$ & $1.8071 e-03$ \\
\hline$E^{N}$ & $4.3729 e-02$ & $2.4999 e-02$ & $1.3452 e-02$ & $7.0021 e-03$ & $3.5755 e-03$ & $1.8071 e-03$ \\
\hline$R^{N}$ & 0.8067 & 0.8940 & 0.9420 & 0.9696 & 0.9845 & - \\
\hline
\end{tabular}

TABle 8: Maximum absolute error of Example 4 after Richardson extrapolation for $\delta=0.6 \varepsilon$ and $\eta=0.5 \varepsilon$.

\begin{tabular}{|c|c|c|c|c|c|c|}
\hline$\varepsilon \downarrow$ & $N \longrightarrow 8$ & 16 & 32 & 64 & 128 & 256 \\
\hline $2^{0}$ & $4.5788 e-03$ & $1.1469 e-03$ & $2.8666 e-04$ & $7.1658 e-05$ & $1.7914 e-05$ & $4.4784 e-06$ \\
\hline $2^{-4}$ & $1.6150 e-02$ & $2.4525 e-03$ & $4.8456 e-04$ & $1.1290 e-04$ & $2.7710 e-05$ & $6.5434 e-06$ \\
\hline $2^{-8}$ & $2.4943 e-02$ & $6.9160 e-03$ & $1.2055 e-03$ & $1.6886 e-04$ & $3.2527 e-05$ & $8.9632 e-06$ \\
\hline $2^{-12}$ & $2.4996 e-02$ & $7.0011 e-03$ & $1.8068 e-03$ & $4.5088 e-04$ & $7.6356 e-05$ & $1.2456 e-05$ \\
\hline $2^{-16}$ & $2.4999 e-02$ & $7.0020 e-03$ & $1.8071 e-03$ & $4.5547 e-04$ & $1.1410 e-04$ & $2.8541 e-05$ \\
\hline $2^{-20}$ & $2.4999 e-02$ & $7.0021 e-03$ & $1.8071 e-03$ & $4.5548 e-04$ & $1.1410 e-04$ & $2.8541 e-05$ \\
\hline $2^{-24}$ & $2.4999 e-02$ & $7.0021 e-03$ & $1.8071 e-03$ & $4.5548 e-04$ & $1.1410 e-04$ & $2.8541 e-05$ \\
\hline $2^{-28}$ & $2.4999 e-02$ & $7.0021 e-03$ & $1.8071 e-03$ & $4.5548 e-04$ & $1.1410 e-04$ & $2.8541 e-05$ \\
\hline $2^{-32}$ & $2.4999 e-02$ & $7.0021 e-03$ & $1.8071 e-03$ & $4.5548 e-04$ & $1.1410 e-04$ & $2.8541 e-05$ \\
\hline $2^{-36}$ & $2.4999 e-02$ & $7.0021 e-03$ & $1.8071 e-03$ & $4.5548 e-04$ & $1.1410 e-04$ & $2.8541 e-05$ \\
\hline $2^{-40}$ & $2.4999 e-02$ & $7.0021 e-03$ & $1.8071 e-03$ & $4.5548 e-04$ & $1.1410 e-04$ & $2.8541 e-05$ \\
\hline$E^{N}$ & $2.4999 e-02$ & $7.0021 e-03$ & $1.8071 e-03$ & $4.5548 e-04$ & $1.1410 e-04$ & $2.8541 e-05$ \\
\hline$R^{N}$ & 1.8360 & 1.9541 & 1.9882 & 1.9971 & 1.9992 & - \\
\hline
\end{tabular}

TABLE 9: Example 3: comparison of maximum absolute error of proposed scheme and result in [15] for $\delta=0.6 \varepsilon$ and $\eta=0.5 \varepsilon$.

\begin{tabular}{|c|c|c|c|c|c|c|}
\hline$\varepsilon \downarrow$ & $N \longrightarrow 8$ & 16 & 32 & 64 & 128 & 256 \\
\hline \multicolumn{7}{|c|}{ Proposed scheme } \\
\hline $10^{-4}$ & $3.0589 e-02$ & $9.4725 e-03$ & $2.5187 e-03$ & $6.3989 e-04$ & $1.6062 e-04$ & $3.9001 e-05$ \\
\hline $10^{-5}$ & $3.0589 e-02$ & $9.4725 e-03$ & $2.5187 e-03$ & $6.3989 e-04$ & $1.6062 e-04$ & $3.9001 e-05$ \\
\hline $10^{-6}$ & $3.0589 e-02$ & $9.4725 e-03$ & $2.5187 e-03$ & $6.3989 e-04$ & $1.6062 e-04$ & $3.9001 e-05$ \\
\hline \multicolumn{7}{|c|}{ Result in [15] } \\
\hline $10^{-4}$ & 0.10207612 & 0.06281638 & 0.03556178 & 0.01906740 & 0.00989895 & 0.00504914 \\
\hline $10^{-5}$ & 0.10210236 & 0.06283382 & 0.03557070 & 0.01907016 & 0.00989794 & 0.00504604 \\
\hline $10^{-6}$ & 0.10210499 & 0.06283557 & 0.03557159 & 0.01907044 & 0.00989798 & 0.00504673 \\
\hline
\end{tabular}

TABle 10: Example 4: comparison of maximum absolute error of proposed scheme and results in [14, 19] for $\delta=0.6 \varepsilon$ and $\eta=0.5 \varepsilon$.

\begin{tabular}{|c|c|c|c|c|c|c|}
\hline \multirow{2}{*}{$\varepsilon \downarrow$} & $N \longrightarrow 10$ & 100 & 10 & 100 & 10 & 100 \\
\hline & \multicolumn{2}{|c|}{ Result in [19] } & \multicolumn{2}{|c|}{ Result in [14] } & \multicolumn{2}{|c|}{ Proposed scheme } \\
\hline $10^{-1}$ & $1.5339 e-02$ & $1.9170 e-04$ & 0.01596700 & 0.00017254 & $9.2052 e-03$ & $5.8912 e-04$ \\
\hline $10^{-2}$ & $2.8175 e-02$ & $1.8656 e-03$ & 0.08016582 & 0.00199505 & $2.0378 e-02$ & $1.0991 e-03$ \\
\hline $10^{-3}$ & $2.8534 e-02$ & $3.3897 e-03$ & 0.10168369 & 0.00999987 & $2.0543 e-02$ & $2.2978 e-03$ \\
\hline $10^{-4}$ & $2.8570 e-02$ & $3.3954 e-03$ & 0.10406804 & 0.01230494 & $2.0554 e-02$ & $2.3059 e-03$ \\
\hline
\end{tabular}

In Figure 1, the influence of the delay parameter on the behaviour of the solution of Examples 3 and 4 is shown for $\varepsilon=2^{-3}$ and $\delta=0.1 \varepsilon, 0.5 \varepsilon$, and $0.9 \varepsilon$. From Figure 2, we observe the numerical solution of Examples 1-4 for different values of the perturbation parameter $\varepsilon=2^{-5}, 2^{-6}$, and $2^{-7}$. As observed in the figures, for $\varepsilon$ going small, strong boundary layer is created. 


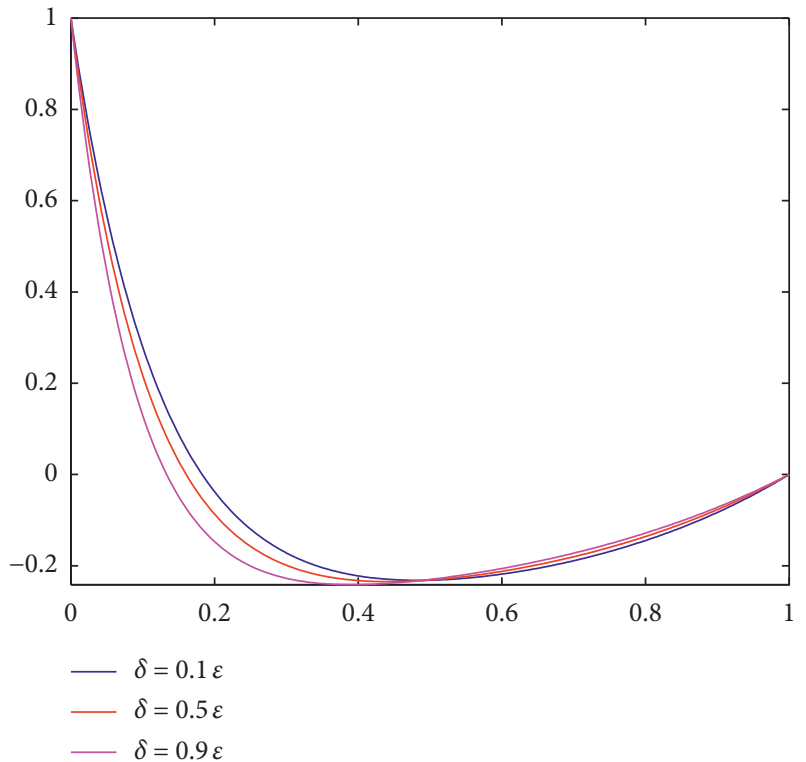

(a)

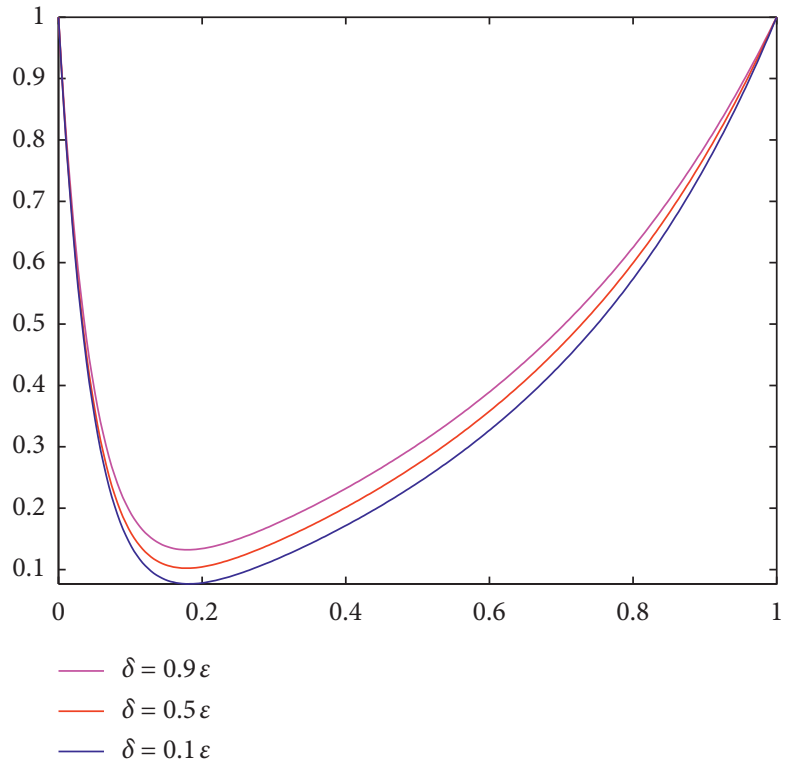

(b)

Figure 1: Effect of delay on the solution behaviour for $\varepsilon=2^{-3}$. (a) Example 3. (b) Example 4.

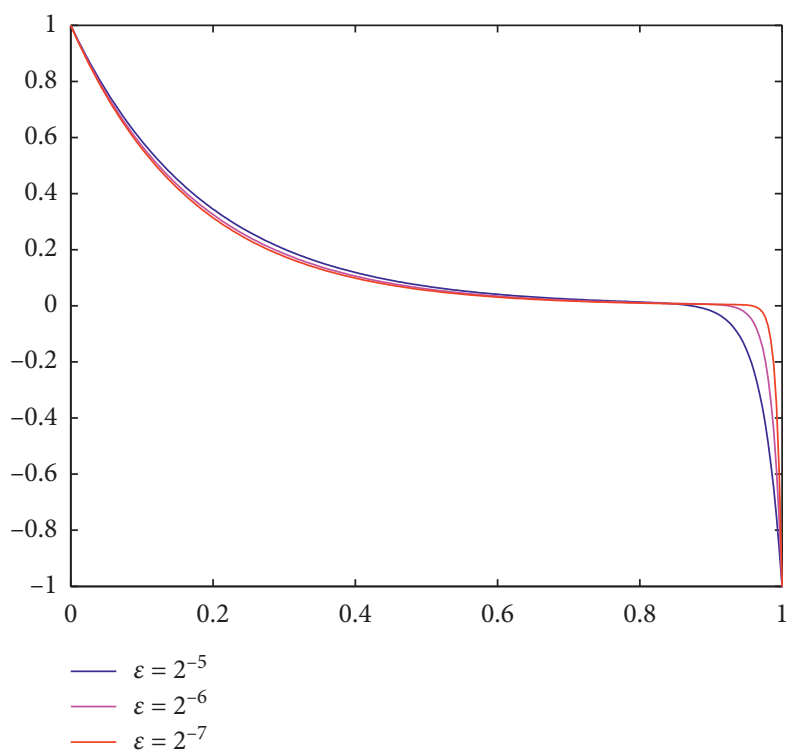

(a)

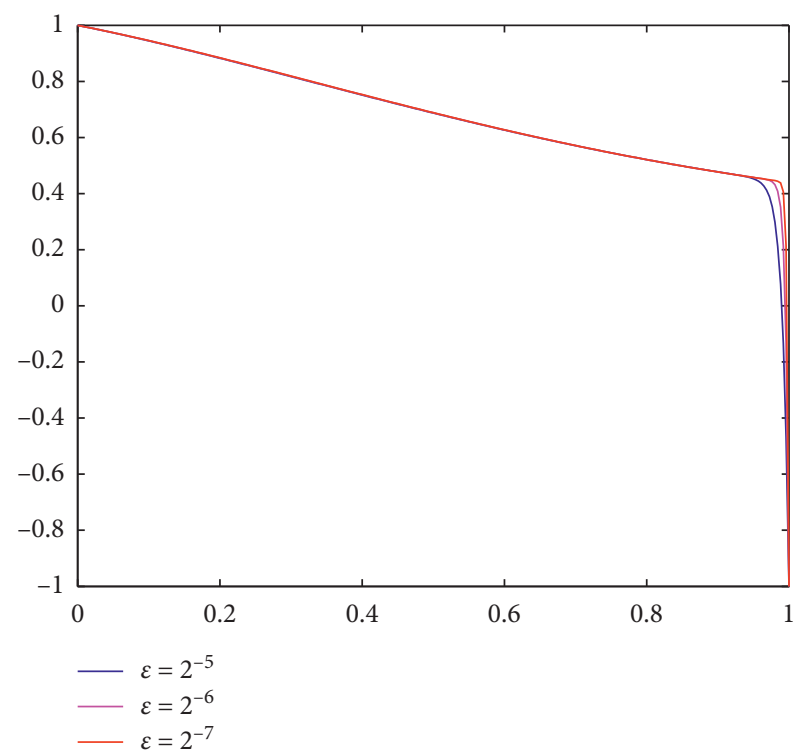

(b)

Figure 2: Continued. 


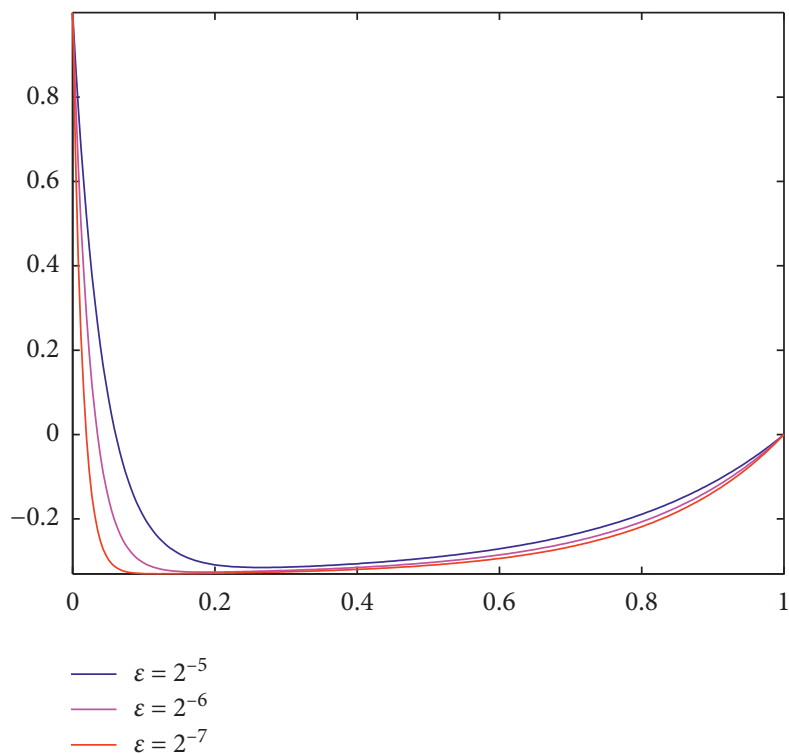

(c)

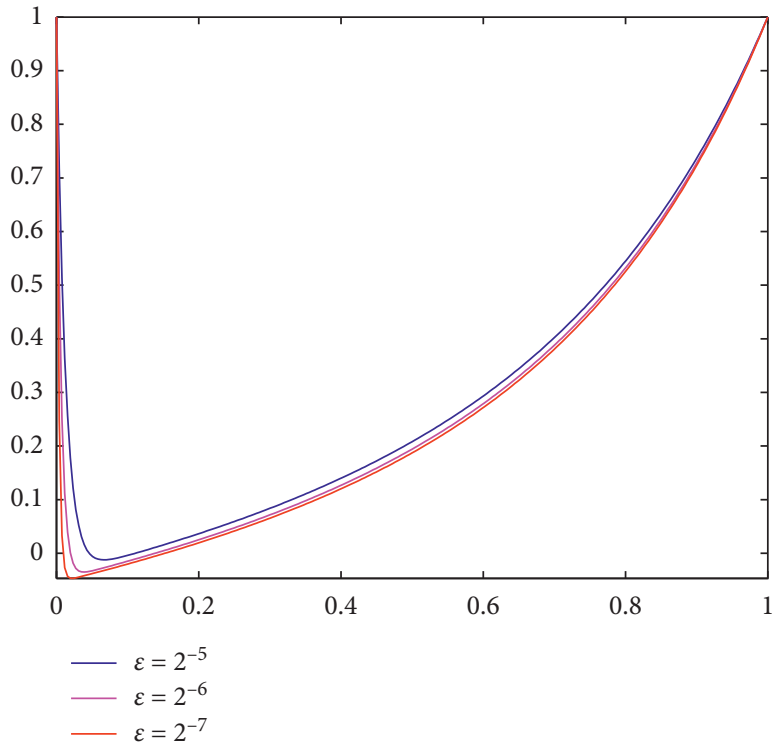

(d)

Figure 2: Boundary layer formation as $\varepsilon$ goes small. (a) Example 1. (b) Example 2. (c) Example 3. (d) Example 4.

\section{Conclusion}

In this paper, singularly perturbed differential difference equations having mixed small shifts on reaction terms of the equation are considered. The considered problem exhibits boundary layer for small values of the perturbation parameter. The bounds and the behaviour of the continuous solution are discussed. Numerical scheme is developed using the technique of exponentially fitted finite difference method. Stability of the scheme is investigated using comparison principle and solution bound. The proposed scheme converges uniformly with rate of convergence of one before Richardson extrapolation and of two after Richardson extrapolation is applied. Test examples exhibiting boundary layers are considered to validate the theoretical finding. The finding in the computation agrees well with the theoretical findings. The proposed scheme gives more accurate results than existing research findings in the literature. In future works, we extend the proposed scheme for singularly perturbed parabolic differential difference equations and singularly perturbed problems with degenerate coefficients.

\section{Data Availability}

No data were used to support the study.

\section{Conflicts of Interest}

The authors declare no conflicts of interest.

\section{References}

[1] Y. Benia, M. Ruggieri, and A. Scapellato, "Exact solutions for a modified Schrödinger equation," Mathematics, vol. 7, no. 10, p. 908, 2019.
[2] Y. Benia and A. Scapellato, "Existence of solution to Korteweg-de Vries equation in a non-parabolic domain," Nonlinear Analysis, vol. 195, p. 111758, 2020.

[3] V. P. Ramesh and M. K. Kadalbajoo, "Upwind and midpoint upwind difference methods for time-dependent differential difference equations with layer behavior," Applied Mathematics and Computation, vol. 202, no. 2, pp. 453-471, 2008.

[4] V. Y. Glizer, "Asymptotic analysis and solution of a finitehorizon $H^{\infty}$ control problem for singularly-perturbed linear systems with small state delay," Journal of Optimization Theory and Applications, vol. 117, no. 2, pp. 295-325, 2003.

[5] R. B. Stein, "Some models of neuronal variability," Biophysical Journal, vol. 7, no. 1, pp. 37-68, 1967.

[6] G. F. Duressa and Y. N. Reddy, "Domain decomposition method for singularly perturbed differential difference equations with layer behavior," International Journal Of Engineering \& Applied Sciences, vol. 7, no. 1, p. 86, 2015.

[7] C. G. Lange and R. M. Miura, "Singular perturbation analysis of boundary value problems for differential-difference equations III. Turning point problems," SIAM Journal on Applied Mathematics, vol. 45, no. 5, pp. 708-734, 1985.

[8] C. G. Lange and R. M. Miura, "Singular perturbation analysis of boundary value problems for differential-difference equations," SIAM Journal on Applied Mathematics, vol. 42, no. 3, pp. 502-531, 1982.

[9] C. G. Lange and R. M. Miura, "Singular perturbation analysis of boundary value problems for differential-difference equations. V. Small shifts with layer behavior," SIAM Journal on Applied Mathematics, vol. 54, no. 1, pp. 249-272, 1994.

[10] C. G. Lange and R. M. Miura, "Singular perturbation analysis of boundary-value problems for differential-difference equations. VI. Small shifts with rapid oscillations," SIAM Journal on Applied Mathematics, vol. 54, no. 1, pp. 273-283, 1994.

[11] M. K. Kadalbajoo, K. C. Patidar, and K. K. Sharma, " $\varepsilon$-Uniformly convergent fitted methods for the numerical solution of the problems arising from singularly perturbed 
general DDEs," Applied Mathematics and Computation, vol. 182, no. 1, pp. 119-139, 2006.

[12] M. K. Kadalbajoo and K. K. Sharma, "Numerical analysis of boundary-value problems for singularly-perturbed differential-difference equations with small shifts of mixed type," Journal of Optimization Theory and Applications, vol. 115, no. 1, pp. 145-163, 2002.

[13] M. K. Kadalbajoo and K. K. Sharma, "Numerical treatment of a mathematical model arising from a model of neuronal variability," Journal of Mathematical Analysis and Applications, vol. 307, no. 2, pp. 606-627, 2005.

[14] M. K. Kadalbajoo and K. K. Sharma, "An $\varepsilon$-uniform convergent method for a general boundary-value problem for singularly perturbed differential-difference equations: small shifts of mixed type with layer behavior," Journal of Computational Methods in Sciences and Engineering, vol. 6, no. 1-4, pp. 39-55, 2006.

[15] D. K. Swamy, K. Phaneendra, and Y. N. Reddy, "Solution of singularly perturbed differential difference equations with mixed shifts using Galerkin method with exponential fitting," Chinese Journal of Mathematics, vol. 2016, Article ID 1935853, 10 pages, 2016.

[16] D. K. Swamy, K. Phaneendra, and Y. N. Reddy, "Accurate numerical method for singularly perturbed differential difference equations with mixed shifts," Khayyam Journal of Mathematics, vol. 4, no. 2, pp. 110-122, 2018.

[17] D. K. Swamy, K. Phaneendra, and Y. N. Reddy, "A fitted non standard finite difference method for singularly perturbed differential difference equations with mixed shifts," Journal de Afrikana, vol. 3, no. 4, 2016.

[18] W. G. Melesse, A. A. Tiruneh, and G. A. Derese, "Solving linear second-order singularly perturbed differential difference equations via initial value method," International Journal of Differential Equations, vol. 2019, Article ID 5259130, 2019.

[19] R. Ranjan and H. S. Prasad, "A novel approach for the numerical approximation to the solution of singularly perturbed differential-difference equations with small shifts," Journal of Applied Mathematics and Computing, 2020, In press.

[20] L. Sirisha, K. Phaneendra, and Y. N. Reddy, "Mixed finite difference method for singularly perturbed differential difference equations with mixed shifts via domain decomposition," Ain Shams Engineering Journal, vol. 9, no. 4, pp. 647-654, 2018.

[21] H. Tian, "The exponential asymptotic stability of singularly perturbed delay differential equations with a bounded lag," Journal of Mathematical Analysis and Applications, vol. 270, no. 1, pp. 143-149, 2002.

[22] J. H. Miller, E. O’Riordan, and G. I. Shishkin, Fitted Numerical Methods for Singular Perturbation Problems: Error Estimates in the Maximum Norm for Linear Problems in One and Two Dimensions, World Scientific, Singapore, 2012.

[23] R. B. Kellogg and A. Tsan, "Analysis of some difference approximations for a singular perturbation problem without turning points," Mathematics of Computation, vol. 32, no. 144, p. $1025,1978$.

[24] R. E. O'Malley, Singular Perturbation Methods for Ordinary Differential Equations, Springer, Berlin, Germany, 1991.

[25] MM. Woldaregay and G. F. Duressa, "Parameter uniform numerical method for singularly perturbed differential difference equations," Journal of the Nigerian Mathematical Society, vol. 38, pp. 223-245, 2019.

[26] D. A. Turuna, M. M. Woldaregay, and G. F. Duressa, "Uniformly convergent numerical method for singularly perturbed convection-diffusion problems," Kyungpook Mathematical Journal, vol. 60, no. 3, 2020.

[27] M. M. Woldaregay and G. F. Duressa, "Fitted numerical scheme for singularly perturbed differential equations having two small delays," Caspian Journal of Mathematical Sciences (CJMS), 2020, In press.

[28] K. Kumar, P. Pramod Chakravarthy, H. Ramos, and J. VigoAguiar, "A stable finite difference scheme and error estimates for parabolic singularly perturbed PDEs with shift parameters," Journal of Computational and Applied Mathematics, 2020, In press. 